\title{
Haemodynamic analysis of adult patients with moyamoya disease: CT perfusion and DSA gradings
}

\author{
Zhiyong Shi (D) , ${ }^{1}$ Guofeng Ma, ${ }^{2}$ Dong Zhang ${ }^{1}$
}

To cite: Shi Z, Ma G, Zhang D. Haemodynamic analysis of adult patients with moyamoya disease: CT perfusion and DSA gradings. Stroke \& Vascular Neurology 2021;6: e000317. doi:10.1136/svn-2019-000317

- Additional material is published online only. To view please visit the journal online (http://dx.doi.org/10.1136/svn2019-000317)

Received 26 December 2019 Revised 10 April 2020 Accepted 2 July 2020 Published Online First 3 September 2020
Check for updates

(C) Author(s) (or their employer(s)) 2021. Re-use permitted under CC BY-NC. No commercial re-use. See rights and permissions. Published by BMJ.

${ }^{1}$ Department of Neurosurgery, Beijing Tiantan Hospital, Capital Medical University, Beijing,

China

${ }^{2}$ Department of Radiology, Beijing Tiantan Hospital, Capital Medical University, Beijing, China

Correspondence to Dr Dong Zhang; zhangdong0660@aliyun.com

\section{ABSTRACT}

Object Moyamoya disease (MMD) is a common and chronic progressive stenotic-occlusive cerebrovascular disease in Eastern Asia. To evaluate the hemispheric haemodynamic status of adult patients with MMD, we explored the potential risk factors of hemispheric perfusion alterations with CT perfusion (CTP) and DSA.

Methods We retrospectively reviewed 44 male and 44 female (176 hemispheres) adult patients with MMD who had both DSA and CTP. Data on cerebral blood perfusion (CBF), cerebral blood volume (CBV), mean transmit time (MTT), time to peak (TTP) of cerebral hemisphere and cerebellum were gathered and difference of relative haemodynamic parameters between different subgroups were assessed with independent sample $t$ analysis, oneway analysis of variance and general linear regression analysis.

Results Parameters in regional CBF (rCBF) of frontal, temporal lobe and basal ganglia in female was more superior than male. rCBF, regional MTT (rMTT) and regional TTP (rTTP) in adult MMD patients with haemorrhage were superior than the ischaemic. With the increase of age, significant difference could be seen in rCBF and rCBV of thalamus. However, with progress of arterial stenosis, significant difference could only be obsevrved in rCBV, rMTT and rTTP, whereas rCBF had no significant difference. For increase of moyamoya vessels, significant decrease of rCBF could be seen in temporal and parietal lobe. With the increase of compensatory artery numbers, no significant difference could be seen in rCBF parameters ( $p>0.05$ ). Conclusions In adult MMD patients, age, gender and clinical type were potential risk factors for the change of cerebral perfusion. When arterial stenosis is worsened, moyamoya vessels could alter perfusion of temporal and parietal lobe, but not frontal lobe. Extracranial/intracranial compensatory arteries could maintain microcirculation stability in frontal lobe and basal ganglia, indicating that the protection from extracranial compensatory arteries, a theoretic base for surgery treatment if necessary.

\section{INTRODUCTION}

Moyamoya disease (MMD) is a common chronic progressive stenotic-occlusive cerebrovascular disease in East Asia. It is characterised by the stenosis of internal carotid artery (ICA) termination and the formation of net-like vessels at the base of brain. ${ }^{12}$ The development of moyamoya vessels or collateral vessels provided an alternative route for the compromised cerebral perfusion. ${ }^{34}$ However, such compensation is often insufficient or unbalanced, resulting in various ischaemic or haemorrhagic strokes. ${ }^{5-7}$ There were several reports concerning that paediatric patients with MMD had a lower cerebral blood perfusion (CBF) compared with agematched controls. ${ }^{8-12}$ Unlike in paediatric MMD patients, the haemodynamic alterations in adult patients has not been well deciphered. ${ }^{1314}$ Previous literature reported that CT perfusion (CTP) was an effective and readily accessible examination to assess the cerebral haemodynamic status and widely used in the clinical practice. ${ }^{15-17}$ Moreover, there was often a disparity of the correlation of hemispheric perfusion with angiographic findings, age, gender and onset types. In our study, we aimed to evaluate the hemispheric haemodynamic status for adult patients with MMD and explore the potential risk factor of hemispheric perfusion alterations by CTP and DSA .

\section{METHODS}

\section{Selection of patients with MMD}

This is a retrospective review. Data of patients diagnosed with MMD from 1 September 2018 to 30 June 2019 were reviewed. The inclusion criteria of our study included: (1) MMD diagnosed according to the guideline ${ }^{18}$ for MMD (criteria of the Research Committee on Spontaneous Occlusion of the Circle of Willis, 2012), (2) age between 18 and 70 years old, (3) completed both CTP and DSA examinations, (4) no history of revascularisation interventions, (5) no cerebral infarctions (based on CT images). Exclusive criteria were: (1) middle cerebral artery (MCA) occlusion, (2) age under 18 or over than 70 years old, (3) obvious brain infarction on CT, (4) history of head surgery and (5) stroke within 1 month prior to the start of the study. All patients with MMID enrolled in our study were required to take both digital subtraction angiography (DSA) and CTP examinations. A total of 88 
Table 1 Information of clinical data and DSA gradings of adult patients with MMD

\begin{tabular}{|c|c|c|c|c|}
\hline Variables & Male $(n=44)$ & Female $(n=44)$ & $\mathrm{T} / \chi^{2} / \mathrm{z}$ & $P$ value \\
\hline Age (year) & $37.95 \pm 8.89$ & $37.77 \pm 8.46$ & 0.098 & 0.922 \\
\hline Clinical type & & & 0.917 & 0.338 \\
\hline Ischaemic & 30 & 34 & & \\
\hline Haemorrhagic & 14 & 10 & & \\
\hline Arteral stenosis & & & 0.487 & 0.487 \\
\hline Mild & 6 & 6 & & \\
\hline Severe & 19 & 23 & & \\
\hline Occlusion & 19 & 15 & & \\
\hline Compensatory srtery & & & 0.027 & 0.868 \\
\hline $0-3$ & 13 & 18 & & \\
\hline $4-6$ & 27 & 19 & & \\
\hline $7-10$ & 4 & 7 & & \\
\hline Moyamoya vessels & & & 0.246 & 0.620 \\
\hline None & 11 & 15 & & \\
\hline Sparse & 23 & 16 & & \\
\hline Dense & 10 & 13 & & \\
\hline
\end{tabular}

Comparion of arterial stenosis, compensatory artery and moyamoya vessels between male and female was set at the hemisphere with advanced Suzuki Stage. $\mathrm{P}<0.05$ represented statistical significance. MMD, moyamoya disease.

patients (44 male and 44 female, 176 hemispheres) met the inclusion criteria and their imaging findings were analysed and shown in table 1 .

\section{Imaging and grading protocol}

DSA assessment in each hemisphere

Moyamoya vessel grades

Moyamoya vessels from ICA (moyamoya-ICA). According to the extent of 'puff smoke' in the brain, moyamoya vessel from ICA could be divided into three grades and scored as: (1) none, no obvious smoke appearance vessel; (2) sparse, smoke appearance vessels formation at the base of brain, but more sparse; (3) dense, large number of smoke appearance vessels anastomosed to form a network at the end of the ICA and expanded in all directions at the base of brain. ${ }^{19}$

\section{Intracranial artery stenosis grades}

According to the classification of Suzuki stages, stenosis degree could be divided into three grades: (1) mild stenosis, those patients with Suzuki I; (2) severe stenosis, those patients with MMD with a Suzuki II and III; (3) occlusion, those patients corresponding to a Suzuki IV, $\mathrm{V}$ and VI. ${ }^{20}$

\section{Grades of intracranial/extracranial compensatory artery}

1. The intracranial arteries included OA, ACA, MCA, anterior choroidal artery, posterior choroidal artery, pericallous artery and posterior cerebral artery (PCA). Extracranial artery included superficial temporal artery, middle meningeal artery (MMA) and occipital artery (OcciA). The maximum number in compensatory arteries evaluated was 10 .

2. When compensatory arterial numbers were between $0-3,4-6$ and $7-10$, they would be classified into group 1 , group 2 and group 3 , respectively.

\section{CTP examination}

A General Electric LightSpeed VCT scanner was used for evaluation of all qualified patients by CTP. The mean time interval from CTP scanning to DSA assessments was $3 \pm 1$ days. Two independent neuroradiologists jointly analysed the CTP data using Neuro CT aw4.7 software (Siemens Medical System) on a Siemens Medical System workstation. Regions of interest (ROIs) in brain were marked with a circle of $32-42 \mathrm{~mm}^{2}$. Vertebral artery (ROI 1) and superior saggital sinus (ROI 2) were set as input artery and output vein respectively. Data and images of cerebral blood volume (CBV) and CBF, mean transit time (MTT) and time-to-peak (TTP) were gathered. In cerebellum reference (ROI 3) was selected at the level of dentate nucleus. The CTP image data sets were displayed side by side, and ROIs were drawn at corresponding positions in both images. ${ }^{16}$

\section{ROI selection (similar to Alberta Stroke program early CT score (ASPECT) scale)}

For individuals, the ROIs at the levels of thalamus and centrum semiovale were as follows (figure 1): superior frontal gyrus (ROI 4 and 14), inferior frontal gyrus (ROI 5 and 15), postcentral gyrus (ROI 6 and 16), supramarginal gyrus (ROI 7 and 17), occipital lobe (ROI 8 and 


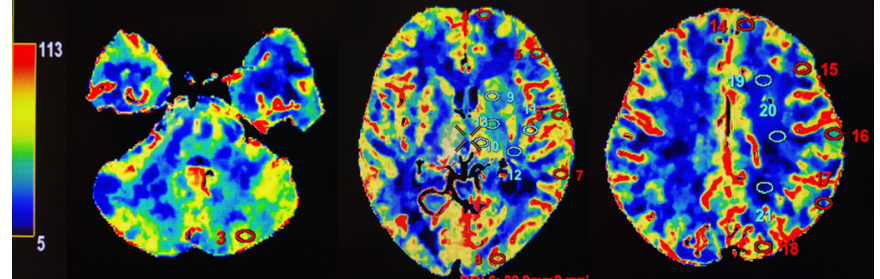

Figure 1 ROI selection (similar to ASPECT scale) ROI 1 was vertebral artery (input artery) and ROI 2 was superior sagittal sinus (output vein). ROI 3 was set in cerebellum at level of dentatum nucleus. ROls from 4 to 18 were as follows: superior frontal gyrus (ROI 4 and 14), inferior frontal gyrus (ROI 5 and 15), postocentral gyrus (ROI 6 and 16), supramarginal gyrus (ROI 7 and 17), occipital lobe (ROI 8 and 18), caudate nucleus (ROI 9), lenticular nucleus, external and internal capsule (ROI 10, 11 and 12) and thalamus (ROI 13). Anterior, central and posterior parts of centrum semiovale were established as ROI 19, 20 and 21. ROI, region of interest.

18), caudate nucleus (ROI 9), lenticular nucleus and internal capsule (ROI 10, 11 and 12) and thalamus (ROI 13). Anterior, central and posterior parts of centrum semiovale were established as ROI 19, 20 and 21. All of these ROIs were mirrored on the contralateral side and represented as mirrored ROIs from 4 to 20 . The data of all these ROIs were calculated and represented as relative values. Means of ROI 3 and mirrored ROI 3 were compared. Mean relative value of ROIs in 4, 5, 14, 15 were frontal lobe and 6,16 were from parietal lobe. Mean relative values of ROIs in 7, 17 represented temporal lobe and 8,18 was occipital lobe. Mean relative values of ROIs in 9, 10, 11 and 12 were basal ganglia and 19, 20 and 21 were centrum semiovale. ${ }^{21}$

\section{Treatment of no perfusion area for haemorrhagic MMD patients}

As for the treatment of no perfusion area, we tried our best to avoid these perfusion defect area. Sometimes we chose to adjust the location of ROI artificially especially in thalamus and basal ganglia to avoid the no perfusion area and decrease the statistical bias.

\section{Statistical analysis}

In our study, SPSS V.22.0 software (IBM) was used for statistical analysis. For continuous variables including age and CTP parameters, independent sample $t$ analysis and one-way analysis of variance (ANOVA) were used as univariate analysis was to seek for the differences between subgroups. For categorised variables including clinical type, $\mathrm{X}^{2}$ analysis was used and if any value was less than 5 , Fisher's exact test was recommended. For rank variables including arterial stenosis, moyamoya vessels and compensatory artery numbers, Mann-Whitney U test was used. Differences were considered to be significant when the statistical $p<0.05$. For comparison of one-way ANOVA between subgroups, post hoc multiple comparisons and Bonferroni correction were used. For the variables with significant statistical differences in univariate analysis $(\mathrm{p}<0.05)$, and the factors that might have significant influence on cerebral perfusion of adult patients with MMD according to our clinical experience, such as gender, age and onset symptoms, general linear regression with Enter model was applied to multivariate. The $\mathrm{P}$ value, the ratio of Odds (OR) and the $95 \%$ CI of the ratio were calculated.

\section{RESULTS \\ Difference in cerebral perfusion between gender, age and clinical type}

The data on all of cerebral perfusion were compared between gender and clinical type. Significant difference was observed between gender and clinical type, with better performance in female patients with haemorrhagic $(\mathrm{p}<0.05)$ (figure 2 , online supplementary material I). With the increase of age, significant difference could been seen in regional CBF (rCBF) of thalamus and regional TTP $($ rTTP) of basal ganglia $(\mathrm{p}<0.05)$ (figure 3 , online supplementary material II).

\section{Difference in brain perfusion between arterial stenosis,} moyamoya vessel and compensatory artery

With the increase of arterial stenosis, significant difference was observed between subgroups: decreased rCBF, increased rCBV, prolonged regional MTT (rMTT) and rTTP $(\mathrm{p}<0.05)$ (figure 3 , online supplementary material II). However, for moyamoya vessels, significant difference was observed in microcirculation, especially in rMTT and rTTP. In addition, with increased compensatory artery numbers, significant decrease in rCBV, rMTT and rTTP could be seen $(\mathrm{p}<0.05)$ (figure 4 , online supplementary material III).

General linear regression between cerebral perfusion and gender, age, clinical type, stenosis, moyamoya vessel, compensatory artery.

For multivariate analysis, female was more superior than male in rCBF of frontal (OR $(95 \%$ CI) $0.243(0.023$ to $0.259), \mathrm{p}=0.02)$, temporal lobe (OR (95\% CI) 0.239 (0.033 to 0.366$), \mathrm{p}=0.019)$ and basal ganglia (OR (95\% CI) 0.295 (0.039 to 0.227$), \mathrm{p}=0.006)$. Parameters of rCBF, rMTT and rTTP of adult MMD patients with haemorrhage were superior than those with ischaemia $(p<0.05)$. With the increase of age, significant difference was seen in $\mathrm{rCBF}$ (OR $(95 \%$ CI) $0.29(0.022$ to $0.148, \mathrm{p}=0.009))$ and $\mathrm{rCBV}$ (OR (95\% CI) 0.237 (0.012 to 0.168$), \mathrm{p}=0.024)$ of thalamus. However, from the view of arterial stenosis, significant difference could only be observed in rCBV, rMTT and rTTP $(p<0.05)$, whereas $\mathrm{rCBF}$ had no significant difference $(p>0.05)$. For moyamoya vessels, significant decrease of $\mathrm{rCBF}$ could be seen in temporal region(OR $(95 \% \mathrm{CI})-0.277(-0.285$ to -0.027$), \mathrm{p}=0.018)$ and parietal lobe (OR (95\% CI) $-0.241(-0.222$ to -0.001$), \mathrm{p}=0.047)$. With the increase of compensatory artery numbers, no significant difference could be seen in rCBF parameters ( $>0.05)$ (figure 5). 


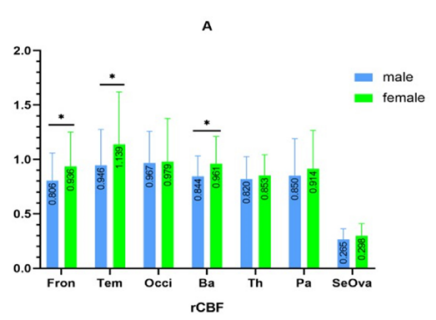

$c$

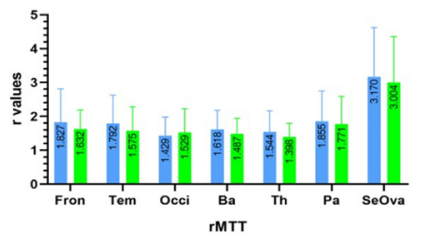

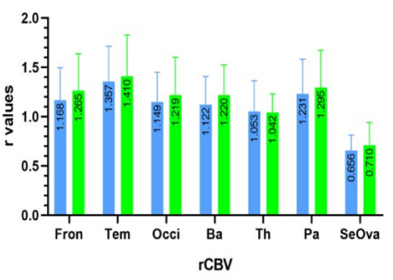

$\mathrm{D}$

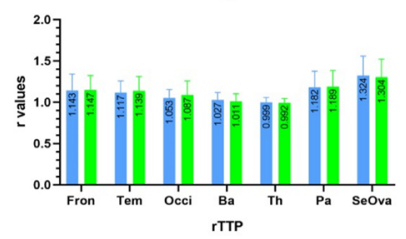

A

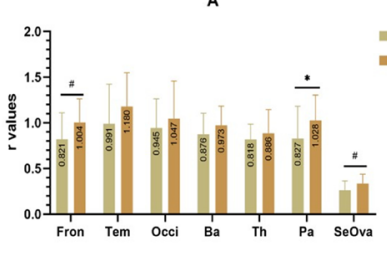

c

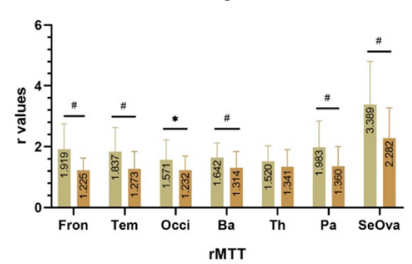

Figure 2 Univariate analysis of haemodynamic condition between gender and clinical type. Figure I and II were the relative value of rCBF, rCBV, rMTT and rTTP. The data of cerebellum at dentate nucleus on parameters of CBF, CBV, MTT and TTP were $61.51 \mathrm{~mL} /(\min \cdot 100 \mathrm{~g}), 2.74 \mathrm{~mL} /(\mathrm{min} \cdot 100 \mathrm{~g}), 2.86 \mathrm{~s}$ and $10.15 \mathrm{~s}$, respectively. ${ }^{*} \mathrm{P}<0.05$; \#P<0.01. (Details could be seen in online supplementary material - figure 1). Ba, basal ganglia; CBF, cerebral blood flow; CBV, cerebral blood volume; Fron, frontal lobe; MTT, mean transit time; Occi, occipital lobe; Pa, parietal lobe; SeOva, centrum semiovale; Tem, temporal lobe; Th, thalamus; TTP, time to peak.

\section{DISCUSSION}

MMD is a chronic progressive disease, with clinical manifestations in different stages of vascular stenosis or cerebral perfusion. ${ }^{2}$ From qualitative analysis, Yin et al reported that hemispheric perfusion status in ischaemia was different from haemorrhage, ${ }^{15}$ indicating that the haemorrhagic had shorter developing history than the ischaemic. Results of our study was consistent with the previous report, which showed perfusion was better in the haemorrhagic group than in the ischaemic group. Moreover, previous literature reported that the ratio of $\mathrm{CBF} / \mathrm{CBV}$ was considered an index of perfusion pressure and the reflection of cerebral circulatory transit time and decreased ratios of $\mathrm{CBF} / \mathrm{CBV}$ were indicative of a prolonged circulation time in MMD. ${ }^{22}$ In our study, value of $\mathrm{rCBF}$ in the haemorrhagic was higher than in ischaemia, and in the contrary $\mathrm{rCBV}$ in haemorrhage was lower than ischaemia, thus causing the microcirculatory parameters of rMTT and rTTP in the ischaemic to be longer than in the haemorrhagic. Some previous studies also reported that $\mathrm{CBF}$ and CVR were not impaired in moyamoya patients with haemorrhagic onset. ${ }^{132324}$ Adult patients with haemorrhage had higher brain perfusion than those with ischaemia, indicating different haemodynamic processes they experienced before onset of symptom. Differences in the extent of hypoperfusion in MMD patients with different clinical type should be noticed.

Our study also found a difference in brain perfusion by different gender, especially $\mathrm{rCBF}$ in frontal, temporal lobe and basal ganglia. However, value of rCBV in female was higher than male. We previously assessed cognitive
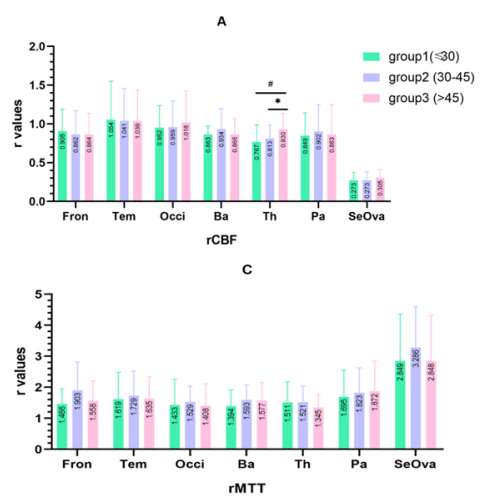
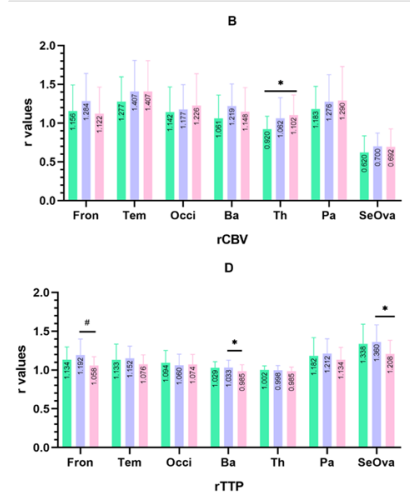
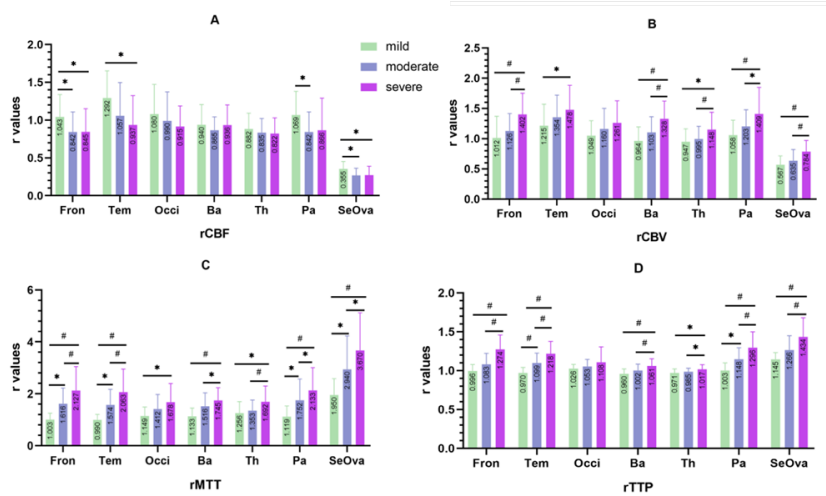

Figure 3 Univariate analysis of haemodynamic condition between age and degree of arterial stenosis. Figure III and IV were the relative value of $\mathrm{rCBF}, \mathrm{rCBV}$, rMTT and $\mathrm{rTTP}(\mathrm{A}-\mathrm{D})$. The data of cerebellum at dentate nucleus on parameters of CBF, CBV, MTT and TTP were $61.51 \mathrm{~mL} /(\mathrm{min} \cdot 100 \mathrm{~g}), 2.74 \mathrm{~mL} /(\mathrm{min} \cdot 100 \mathrm{~g}), 2.86 \mathrm{~s}$ and $10.15 \mathrm{~s}$ respectively. ${ }^{*} \mathrm{P}<0.05$; \#P<0.01. (Details could be seen in online supplementary materials - figure 2). Ba, basal ganglia; CBF, cerebral blood flow; CBV, cerebral blood volume; Fron, frontal lobe; MTT, mean transit time; Occi, occipital lobe; Pa, parietal lobe; SeOva, centrum semiovale; Tem, temporal lobe; Th, thalamus; TTP, time to peak. 

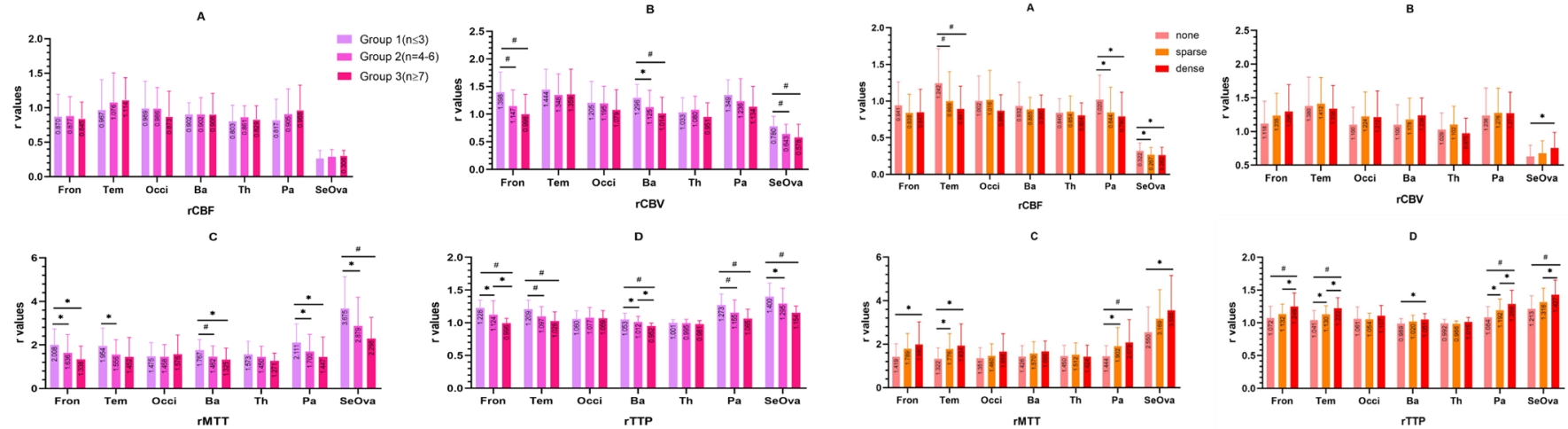

Figure 4 Univariate analysis of haemodynamic condition between compensatory artery numbers and moyamoya vesses. Figure $\mathrm{V}$ and $\mathrm{VI}$ were the relative value of $\mathrm{rCBF}, \mathrm{rCBV}$, rMTT and $\mathrm{rTTP}(\mathrm{A}-\mathrm{D})$. The data of cerebellum at dentate nucleus on parameters of CBF, CBV, MTT and TTP were $61.51 \mathrm{~mL} /(\mathrm{min} \cdot 100 \mathrm{~g}), 2.74 \mathrm{~mL} /(\mathrm{min} \cdot 100 \mathrm{~g}), 2.86 \mathrm{~s}$ and $10.15 \mathrm{~s}$, respectively. ${ }^{\star} \mathrm{P}<0.05$; $\# \mathrm{P}<0.01$. (Details could be seen in online supplementary materials - figure 3 ). Ba, basal ganglia; CBF, cerebral blood flow; CBV, cerebral blood volume; Fron, frontal lobe; MTT, mean transit time; Occi, occipital lobe; Pa, parietal lobe; SeOva, centrum semiovale; Tem, temporal lobe; Th, thalamus; TTP, time to peak.

function of 49 adult patients with MMD and found female prospective memory and attention was better than male, ${ }^{25}$ a finding partially supported by our haemodynamic assessment. Hara et al recognised that oestrogen facilitated higher cognitive functions by enhancing the prefrontal cortex and hippocampus area. ${ }^{26}$ We also hypothesised that haemodynamic difference in gender existed in different brain region, especially in cognition related brain areas. In our research, perfusion of hippocampus and prefrontal cortex were better in female than male. As for the relationship between perfusion and oestrogen, we hypothesised that oestrogen played a protective role in preserving haemodynamic circulation and cognitive function, which needed to be explored further.

Normal CBF was known to correlate negatively with age. ${ }^{27}$ Previous literature reported that with the progress of MMD, long duration of ischaemia, loss of neuronal mass, atherosclerotic disease, diabetes and hypertension might also play a role in affecting cerebral haemodynamics, thus causing a reduction in metabolic demand for adult patients with MMD. ${ }^{23} 29$ Though no statistical significance could be found in our research, negative correlation between age and rCBF, rTTP could be observed, especially in frontal, temporal and parietal lobes. However, positive correlation in thalamic or basal ganglia regions could be observed, which may explain cerebral bleeding was more common in thalamic than brain cortex. For those MMD treated with conservative options were still exposed to the possibility of brain ischaemia because of the natural process of negative correlation between age and cortical perfusion.

In addition, previous studies had reported that the stenosis of ICA and PCA were closely linked with the prolonged MTT because of low perfusion pressure. ${ }^{30}$ In our research, progress of arterial stenosis was associated with changes of rCBV, rMTT and rTTP, which was
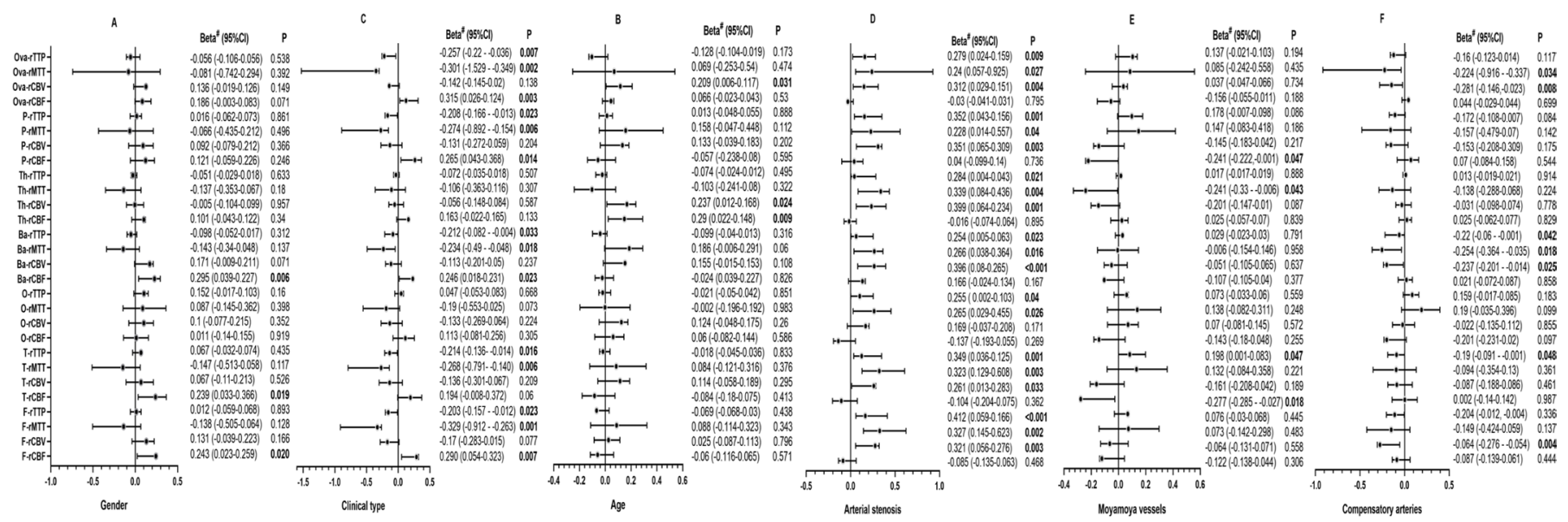

Figure 5 Multivariate analysis of haemodynamic condition between gender, clinical type, age, arterial stenosis, moyamoya vessels and compensatory artery numbers. All data were represented as relative value of rCBF, rCBV, rMTT and rTTP. Beta was represented as standardised coefficients. Ba, basal ganglia; CBF, cerebral blood flow; CBV, cerebral blood volume; Fron, frontal lobe; MTT, mean transit time; Occi, occipital lobe; Pa, parietal lobe; SeOva, centrum semiovale; Tem, temporal lobe; Th, thalamus; TTP, time to peak. 
consistent with previous findings indicating the simultaneous brain ischaemia and compensation. However, no significant association between arterial stenosis and rCBF could be found, suggesting that with the progress of MMD, moyamoya vessels or collateral vessels helped maintain haemodynamic perfusion. Previous papers also reported decreased $\mathrm{CBF}$ in the frontal and temporal regions comparing to occipital lobe and basal ganglia. ${ }^{31}$ In our research, with the progress of arterial stenosis, cerebral perfusion pattern changed, where little disturbance in $\mathrm{CBF}$ of the posterior region and a marked disturbance in CBF of the frontal lobes were observed. We speculated that perfusion of temporal and parietal lobes relied on the vertebrobasilar system and PCA via leptomeningeal collaterals or moyamoya vessels through intraparenchymal anastomoses, whereas the frontal lobe was lacking of such collaterals.

Furthermore, extensive development of basal ganglia moyamoya vessels was a sign of severe haemodynamic impairment, a process accelerated by persistent cortical ischaemia. ${ }^{24}$ Our analsysis had found significant reduction of $\mathrm{rCBF}$ in parietal and temporal lobe in MMD patients with more moyamoya vessels. However, no significant difference in $\mathrm{rCBF}$ was observed between sparse and dense subgroups even significant prolongation of rMTT and rTTP in cortical and basal ganglia regions were seen. We speculated that the appearance of moyamoya vessel was a signal of haemodynamic ischaemia, especially when there was a reduction of $\mathrm{rCBF}$ and prolongation of rMTT and rTTP in parietal and temporal lobe. The extent of moyamoya vessels could partially predict the severity of ischaemia, because of negative correlation between brain cortex and moyamoya vessels, especially in parietal and temporal cortex.

Moreover with the increase of compensatory artery numbers, increased cortical perfusion could not be observed, but negative correlation with rCBV, rMTT, rTTP could be found. Previous literature reported that intracerebral collaterals could not provide adequate blood supply to the cerebral cortex, even if they were fully developed. ${ }^{24}$ However, our research found that for adult MMD patients without obvious cerebral infarction, compensatory arteries were capable of maintaining microcirculation stability in frontal lobe and basal ganglia without improving brain blood perfusion. Furthermore, we hypothesised that haemodynamic appearance of compensatory arteries and disappearence of moyamoya vessels existed simultaneously. This process of getting help from intracranial/ extracranial arterial collaterals indicated the function of extracranial compensatory artery, and potential usefulness if surgery was necessary. Revascularisation could artificially achieved the reconstruction of CBF distribution. The common operation area was mainly concentrated in the parietal rather than frontal lobes. The abundant compensatory channels existing in brain could realise the self-regulation of various areas of $\mathrm{CBF}$.

Our study also had some limitations. First, the study population was small and with no control group was established to compare the unique cerebral haemodynamics. Second, the patients selected into our study were adults patients, so it may not apply to paediatric patients with MMD. Third, the accurate drawing of ROI at the same level and size was difficult. Fourth, it was not enough to evaluate only the number of compensatory vessels but not the degree of compensation and might bring bias. Fifth, cerebral haemodynamics was very complex in MMD. Thereby, it might be arbitrarily to assess single cerebral lobe rather than entire cerebral hemisphere.

\section{CONCLUSIONS}

In adult patients with MMD, age, gender and clinical type were potential risk factors for the change of cerebral perfusion. When arterial stenosis is worsened, moyamoya vessels could alter perfusion of temporal and parietal lobe, but not frontal lobe. Extracranial/intracranial compensatory arteries could maintain microcirculation stability in frontal lobe and basal ganglia, indicating that the protection from extracranial compensatory arteries, a theoretic base for surgery treatment if necessary.

Contributors ZS, first author: design and conceptualised study; analysed the data; drafted the manuscript for intellectual content. DZ, corresponding author: design and conceptualised study; drafting and revision for intellectual content. GM, coauthor: reviewed all protocol and statistical analysis.

Funding The authors have not declared a specific grant for this research from any funding agency in the public, commercial or not-for-profit sectors.

Competing interests None declared.

Patient consent for publication Not required.

Ethics approval Our study was approved by the Ethics Committee of Beijing Tiantan Hospital, China and the ethics number was KY 2019-084-02.

Provenance and peer review Not commissioned; externally peer reviewed. Data availability statement Data are available in a public, open access repository. Our data are not in a repository. Any subscribers can who are interested in our database can send email requests to authors Zhiyong Shi (szy1195156829@aliyun. com). Thanks!

Supplemental material This content has been supplied by the author(s). It has not been vetted by BMJ Publishing Group Limited (BMJ) and may not have been peer-reviewed. Any opinions or recommendations discussed are solely those of the author(s) and are not endorsed by BMJ. BMJ disclaims all liability and responsibility arising from any reliance placed on the content. Where the content includes any translated material, BMJ does not warrant the accuracy and reliability of the translations (including but not limited to local regulations, clinical guidelines, terminology, drug names and drug dosages), and is not responsible for any error and/or omissions arising from translation and adaptation or otherwise.

Open access This is an open access article distributed in accordance with the Creative Commons Attribution Non Commercial (CC BY-NC 4.0) license, which permits others to distribute, remix, adapt, build upon this work non-commercially, and license their derivative works on different terms, provided the original work is properly cited, appropriate credit is given, any changes made indicated, and the use is non-commercial. See: http://creativecommons.org/licenses/by-nc/4.0/.

ORCID iD

Zhiyong Shi http://orcid.org/0000-0003-3392-0041

\section{REFERENCES}

1 Suzuki J, Kodama N. Moyamoya disease--a review. Stroke 1983;14:104-9.

2 Amlie-Lefond C, Zaidat OO, Lew SM. Moyamoya disease in early infancy: case report and literature review. Pediatr Neurol 2011;44:299-302. 
3 Scott RM, Smith ER. Moyamoya disease and moyamoya syndrome. N Engl J Med 2009;360:1226-37.

4 Sasagawa A, Mikami T, Hirano T, et al. Characteristics of cerebral hemodynamics assessed by CT perfusion in moyamoya disease. $J$ Clin Neurosci 2018;47:183-9.

5 Han DH, Nam DH, Oh CW. Moyamoya disease in adults: characteristics of clinical presentation and outcome after encephaloduro-arterio-synangiosis. Clin Neurol Neurosurg 1997;99 Suppl 2:S151-5.

6 Kuroda S, Houkin K. Moyamoya disease: current concepts and future perspectives. Lancet Neurol 2008;7:1056-66.

7 Lee S-B, Kim D-S, Huh P-W, et al. Long-Term follow-up results in 142 adult patients with moyamoya disease according to management modality. Acta Neurochir 2012;154:1179-87.

8 Ikezaki K, Matsushima T, Kuwabara Y, et al. Cerebral circulation and oxygen metabolism in childhood moyamoya disease: a perioperative positron emission tomography study. $J$ Neurosurg 1994;81:843-50.

9 Kazumata K, Kuroda S, Houkin K, et al. Regional cerebral hemodynamics during re-build-up phenomenon in childhood moyamoya disease. An analysis using 99mTc-HMPAO SPECT. Childs Nerv Syst 1996;12:161-5

10 Kuroda S, Houkin K, Kamiyama H, et al. Regional cerebral hemodynamics in childhood moyamoya disease. Childs Nerv Syst 1995;11:584-90.

11 Kuroda S, Kamiyama $\mathrm{H}$, Abe $\mathrm{H}$, et al. Cerebral blood flow in children with spontaneous occlusion of the circle of Willis (moyamoya disease): comparison with healthy children and evaluation of annual changes. Neurol Med Chir 1993;33:434-8.

12 Kuwabara Y, Ichiya Y, Otsuka M, et al. Cerebral hemodynamic change in the child and the adult with moyamoya disease. Stroke 1990;21:272-7.

13 Taki W, Yonekawa Y, Kobayashi A, et al. Cerebral circulation and metabolism in adults' moyamoya disease--PET study. Acta Neurochir 1989;100:150-4.

14 Kuwabara Y, Ichiya Y, Sasaki M, et al. Response to hypercapnia in moyamoya disease. cerebrovascular response to hypercapnia in pediatric and adult patients with moyamoya disease. Stroke 1997;28:701-7.

15 Yin $\mathrm{H}$, Liu X, Zhang D, et al. A novel staging system to evaluate cerebral hypoperfusion in patients with moyamoya disease. Stroke 2018;49:2837-43.

16 Zhou J, Zhang H, Gao P, et al. Assessment of perihematomal hypoperfusion injury in subacute and chronic intracerebral hemorrhage by CT perfusion imaging. Neurol Res 2010;32:642-9.

17 Zhang J, Wang J, Geng D, et al. Whole-Brain CT perfusion and CT angiography assessment of moyamoya disease before and after surgical revascularization: preliminary study with 256 -slice CT. PLOS One 2013;8:e57595.

18 Health Labour Sciences Research Grant for Research on Measures for Intractable Diseases. Guidelines for diagnosis and treatment of moyamoya disease (spontaneous occlusion of the circle of Willis) Neurol Med Chir 2012;52:245-66.

19 Zhao Y, Li J, Lu J, et al. Predictors of neoangiogenesis after indirect revascularization in moyamoya disease: a multicenter retrospective study. J Neurosurg 2019:1-11.

20 Suzuki J, Takaku A. Cerebrovascular "moyamoya" disease. Disease showing abnormal net-like vessels in base of brain. Arch Neurol 1969;20:288-99.

21 Hungerford JP, Hyer M, Turk AS, et al. Impact of aspect scores and infarct distribution on outcomes among patients undergoing thrombectomy for acute ischemic stroke with the adapt technique. $J$ Neurointerv Surg 2017;9:823-9.

22 Gibbs JM, Wise RJ, Leenders KL, et al. Evaluation of cerebral perfusion reserve in patients with carotid-artery occlusion. Lancet 1984;1:310-4.

23 Nariai T, Matsushima Y, Imae S, et al. Severe haemodynamic stress in selected subtypes of patients with moyamoya disease: a positron emission tomography study. J Neurol Neurosurg Psychiatry 2005; 76:663-6.

24 Piao R, Oku N, Kitagawa K, et al. Cerebral hemodynamics and metabolism in adult moyamoya disease: comparison of angiographic collateral circulation. Ann Nucl Med 2004;18:115-21.

25 Shi Z, Wen Y-J, Huang Z, et al. Different aspects of cognitive function in adult patients with moyamoya disease and its clinical subtypes. Stroke Vasc Neurol 2020;5:e000309:86-96.

26 Hara Y, Waters EM, McEwen BS, et al. Estrogen effects on cognitive and synaptic health over the lifecourse. Physiol Rev 2015;95:785-807.

27 Østergaard L. Principles of cerebral perfusion imaging by bolus tracking. J Magn Reson Imaging 2005;22:710-7.

28 Sato H, Sato N, Tamaki N, et al. Chronic low-perfusion state in children with moyamoya disease following revascularization. Childs Nerv Syst 1990;6:166-71.

29 Laborde G, Harders A, Klimek L, et al. Correlation between clinical, angiographic and transcranial Doppler sonographic findings in patients with moyamoya disease. Neurol Res 1993;15:87-96.

30 Togao O, Mihara F, Yoshiura T, et al. Cerebral hemodynamics in moyamoya disease: correlation between perfusion-weighted MR imaging and cerebral angiography. AJNR Am J Neuroradiol 2006;27:391-7.

31 Ogawa A, Yoshimoto T, Suzuki J, et al. Cerebral blood flow in moyamoya disease. Part 1: correlation with age and regional distribution. Acta Neurochir 1990;105:30-4. 